\title{
Knowledge-Based Approach for System Level Electromagnetic Safety Analysis
}

\author{
Lokesh Devaraj \\ Vehicle Resilience Technologies, HORIBA MIRA Limited, UK. E-mail: lokesh.devaraj@horiba-mra.com
}

\author{
Alastair R. Ruddle \\ Vehicle Resilience Technologies, HORIBA MIRA Limited, UK. E-mail: alastair.ruddle@ horiba-mira.com
}

Qazi Mashaal Khan

RF-EMC Group, École Supérieure d'Électronique de l'Ouest, France.E-mail: qazimashaal.khan@eseo.fr

\author{
Alistair P. Duffy \\ Institute of Engineering Sciences, De Montfort University, UK. E-mail: apd@dmu.ac.uk
}

\begin{abstract}
Road vehicles and similarly complex systems are constructed by integrating many subsystems and components that are sourced from a large number of suppliers. This process may lead to the emergence of possible system-level safety issues, some of which could be caused by external or internal electromagnetic interference. Assurance of safety by demonstrating compliance with standard tests is becoming increasingly challenging as system complexity rises. This is due to the costs and practical limitations of both component and system-level electromagnetic compatibility testing. Hence, there is a need for additional methods to help estimate the likelihood of electromagnetic interference risks associated with such systems. Probabilistic graphical models, such as Bayesian and Markov networks, are able to provide a better visualization of various features and their relationships in a single graphical structure. Moreover, using template models, a general-purpose representation for various integrated components of a system can be developed for collective inference. Using such methods, this paper proposes a knowledge-based approach to assist risk management in system-level electromagnetic engineering. The purpose of using a knowledge-based approach is to be able to undertake safety risk analyses during the early stages of design, when many factors (e.g. internal, and external electromagnetic interference levels, physical location of the component) remain uncertain.
\end{abstract}

Keywords: Safety, Bayesian networks, risk analysis, template models, knowledge-based methods, EMC.

\section{Introduction}

Since the advent of cars back in 1886, road vehicles have gone through many remarkable advancements in their design, features, and functionalities. Furthermore, reduced design constraints due to electrification has given rise to possibilities of realizing new system concepts and ideas. However, technological developments that enable such new features also lead to increase in the proportion of electrical and electronic components (some performing safety-critical functions) within a vehicle.

Due to increasing system complexity, the current rule-based electromagnetic engineering approach is considered to be inadequate to ensure achievement of the desired safety and reliability levels Armstrong (2006). By adopting a riskbased approach to electromagnetic compatibility (EMC), critical design decisions (e.g. safetyrelated) can be taken with informed risk levels relating to possible system level hazards. To identify the tools required to support a risk-based systemlevel electromagnetic (EM) approach for func- tional safety, the limitations of traditional tools like fault tree analysis, event tree analysis and bow-tie analysis, and the benefits of probabilistic graphical models (PGMs) like Bayesian Networks (BN) and Markov random fields, are already discussed in Devaraj et al. (2020).

Moreover, recent studies in the field of safety and reliability show a significant increase in the use of BNs for risk assessment, taking advantage of their system modelling capabilities and inference techniques Washington et al. (2019). Additionally, PGM models also allow the combination of expert knowledge and available data for likelihood estimation of desired variables during safety analysis. It is therefore possible to employ these models in the very early stages of system development when firm data is usually scarce.

The application of BNs in a knowledge-based approach to model epistemic uncertainties is discussed in Section 2 of this paper. The model parameters and associated probability values (obtained from either from subjective beliefs or objective simulation data) that are used to construct

Proceedings of the 31th European Safety and Reliability Conference.

Edited by Bruno Castanier, Marko Cepin, David Bigaud and Christophe Berenguer

Copyright $\odot 2021$ by ESREL2021 Organizers. Published by Research Publishing, Singapore

ISBN: 981-973-0000-00-0 :: doi: 10.3850/981-973-0000-00-0_output 
the BN template model for the likelihood estimation of component performance levels are described using a case study in Section 3 . Using examples taken from the case study, Section 4 outlines the Bayesian inference techniques that can be used for both predictive and diagnostic safety analyses. The conclusion of this paper is provided in Section 5

\section{Modelling Epistemic Uncertainties}

For adopting a risk-based safety analysis, it is necessary to estimate the likelihood of all possible hazards caused due to failure or malfunctioning behaviour of safety elements within the system. However, epistemic uncertainties (i.e. those due to lack of system knowledge) pose a major challenge during the risk estimation process. For instance, during vehicle integration some components (software and hardware units) are not designed for the specific system, and hence their exact functional performance with respect to the EM environment of the target system is usually unknown. Estimating the likelihood of EMI causing system-level safety hazards is the primary objective. Nevertheless, for overall system safety, uncertainty due to other system parameters (such as temperature, ageing, vibrations etc.) can also be included.

Graphical models such as BN have been used for applications including system reliability modelling Marquez et al. (2010), safety analysis for remotely piloted aircraft Washington et al. (2019) and automated ships Hanninen and Kujala (2012), and in the management of risk more generally Fenton and Neil (2007). With the BN modelling technique it is possible to describe any acyclic causal relationships between variables using graphical networks comprising nodes and directed edges between them. Each node in the graph represents a variable, whose state space (all possible values a variable can take) can either be discrete or continuous. The directed edges emerging from one node towards another denotes an influential or causal relationship. The nodes which influences other nodes are called the parent nodes, and the ones being influenced are called child nodes. Hence, the probability distribution of every child node variable is conditioned on its parent node variables, and nodes without any parents are conditioned on an empty set. For example, in Fig 1, the node L (representing component location) influences both nodes $\mathrm{E}$ (representing exposure to EMI) and T (exposure to temperature).

In a knowledge-based approach, the process of learning the BN structure is usually done by incorporating expert's knowledge and/or based on intuition of cause-effect relationship. The values in the conditional probability distribution (CPD) tables assigned to each node of the BN denote the belief measure of the system expert. Nevertheless, the structure and the CPD values can also be determined by using various structure learning algorithms given in Koller and Friedman (2007) and the latter when relevant system's statistical data is made available. In addition to the possible combination of data and knowledge for analysing uncertain parameters in a single model, various Bayesian inference techniques that can be applied for such models Devaraj et al. (2021) makes it suitable for predictive and diagnostic safetyanalyses at different stages of the system development process.

\section{Template Models}

We know that, for systems such as road vehicles, the EM environment and other operational conditions usually vary for each component. So, any critical decision that involves safety-related components needs to consider these system uncertainties. Template models are generic PGM structures that can be reused in different scenarios for estimating probability values of system parameters that are included within the model, see SERENE (1999) and Koller and Friedman (2007).

In Fig. 11, the node L (representing component location) influences both nodes E (representing exposure to EMI) and T (reflecting exposure to temperature). This basic template can then be instantiated for different types of component (which are therefore exposed to differing operational environments), as well as for differing options for a specific component of a particular subsystem (which would all be exposed to the same operational environment).

\subsection{Case Study}

The component used for the purposes of the case study is a voltage-controlled oscillator (VCO). VCOs are used in systems that implement a wide variety of functions (such as communication, production of electronic music, function generators etc.). In this case study, a performance comparison is to be undertaken for two such components; a Ring- VCO (denoted V1), and Current-StarvedVCO (V2). The assumption is that one of them is to be chosen for inclusion in a safety-related vehicle function. Provision of the correct output

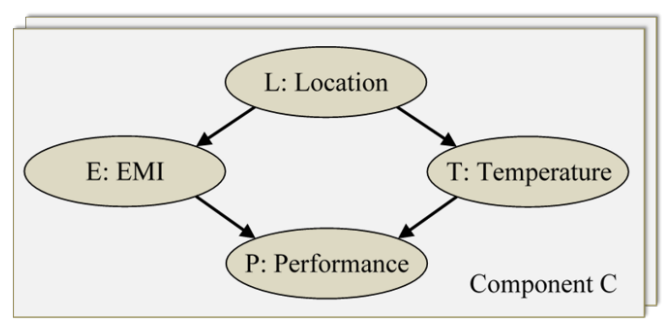

Fig. 1. Component performance template. 
frequency for these devices is assumed to be affected by both the ambient operating temperature and the impact of external EMI noise coupled into the controlling voltage signal.

Within the scope of this case study, the aim is purely to demonstrate the utility of template models for risk management and decision. Consequently, details regarding the design of the two components $\mathrm{V} 1$ and $\mathrm{V} 2$ are not relevant to this argument and are therefore not discussed further.

\subsection{Template Model Parameters}

For the case study, a simple BN template model that can be used to determine the performance of various system components is shown in Fig. 1. The BN model consists of four node variables:

- component location $(L)$ within the vehicle;

- EMI frequency $(F)$ at $L$;

- ambient temperature $(T)$ at $L$;

- component performance $(P)$ at $L$.

\subsubsection{Location}

Initially, the location for packing any component within the vehicle is assumed to be unknown. The vehicle is divided into three zones as illustrated in Fig. 2. L1 (engine bay), L2 (cabin) and L3 (luggage compartment). Probability values for each state of variable $L=L 1, L 2, L 3$ can be assigned by a system expert, usually based on prior knowledge of various kinds (available space for packaging, relationship to other components, styling requirements, location of the component in previous system model etc.). The probability distribution of variable $L$ assumed for the case study is given in Table 1. This distribution represents a possible expert's belief regarding likelihood values for each location within the vehicle.

\subsubsection{EMI Frequency}

The range of EMI frequencies considered for the current investigation is categorized as low $(0.5-10$ $\mathrm{MHz})$, medium (10-100 MHz), and high (0.1-1 $\mathrm{GHz}$ ). Each of these categories is considered as a possible state that can be assigned for the node variable $E=E 1, E 2, E 3$. In the BN template, node

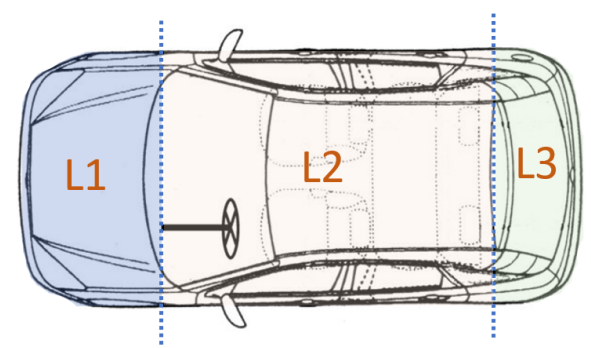

Fig. 2. Component performance template.
$\mathrm{L}$ is assigned as the parent of node $\mathrm{E}(\mathrm{L} \rightarrow \mathrm{E})$ because the probability of EMI noise frequency in the ranges specified by the variable states $E$ differ for the various system locations (denoted by variable $L$ ). For example, in cars of sedan type, the luggage compartment (L3) is often highly shielded by metallic structures, providing relatively higher shielding effectiveness from external EMI when compared to the engine bay (L1) and the cabin space (L2). Due to this dependence, each state space of variable $E$ is conditioned on all possible states of node variable $L$ to derive the CPD table $\operatorname{Pr}(E \mid L)$ shown Table 2 .

For the purpose of the case study, the CPD entries of $\operatorname{Pr}(E \mid L)$ are assigned based on reasonable beliefs an expert might have. For example, the probability of observing EMI noise at the lowest frequencies $(E 1)$ given the cabin location $(L 2)$, the probability $\operatorname{Pr}(E 1 \mid L 2)$ is assigned a very low value of $2 \%$. This is because, due to the dimensions of the windows present in zone L2 of the vehicle, external EM waves with low frequencies are expected to be poorly coupled into the interior. Numerical simulation results reported in Ruddle et al. (2004) support this belief. Similar reasoning can be provided to complete the CPD values for $\operatorname{Pr}(E \mid L 1)$ and $\operatorname{Pr}(E \mid L 2)$. It can be observed that, for the case of $\operatorname{Pr}(E \mid L 3)$, all assignments are considered equally likely, as no additional information is assumed available. Nevertheless, measurements could be carried out, such as those described by Harima et al. (2016), to assign more informed probability values for a specific system.

\subsubsection{Temperature}

The temperature (T) at the component location (L) is considered as an additional system parameter in the template for predicting the component performance. The dependence of component temperature on its location is denoted by the edge $\mathrm{L} \rightarrow \mathrm{T}$. For node variable $T$ the state space includes $T 1$, $T 2$ and $T 3$, which correspond to low $\left(0-24^{\circ} \mathrm{C}\right)$, medium $\left(24-52{ }^{\circ} \mathrm{C}\right)$ and high $\left(52-80{ }^{\circ} \mathrm{C}\right)$ temperatures, respectively. The CPD values of $\operatorname{Pr}(T \mid L)$ used for the case study (see Table 3) are based on typical temperatures for the vehicle zones considered. For instance, in cars with internal combustion engines, the temperatures in zone L1 is usually high, compared to the lower temperatures maintained/observed in zones L2 and L3.

\subsubsection{Performance}

For the model considered here, the performance (P) of a component depends on both the temperature and EMI noise frequency. The nodes $\mathrm{T}$ and $E$ are therefore assigned as parent nodes of node $P$. The three possible states of node variable $P=P 1, P 2, P 3$ correspond to good, moderate, and poor performance levels of the component.

Unlike the previously discussed node variables 
$L, E$ and $T$, which are common parameters for all components of this type, $\mathrm{P}$ is a parameter that is specific to a particular component of that type. Consequently, when the template is used for comparison of multiple component performances, the CPD table of node variable $P$ should be updated for every component, whereas the CPDs associated with the other system parameters remain unchanged.

\section{Application of BN Template Model}

As a case study, the $\mathrm{BN}$ template model discussed in Section 3, is applied for the comparison of performance levels between the components V1 and V2. Both components have similar function of converting a DC input voltage to a sinusoidal output voltage of fixed frequency. However, as these components have different circuit designs, their functional performance differs with respect to the operating temperature and EMI noise frequency present in the system. Functional deviations due

Table 1. CPD table for node $\mathrm{L}$.

\begin{tabular}{cccc}
\hline & $L 1$ & $L 2$ & $L 3$ \\
$\operatorname{Pr}(L)$ & $\begin{array}{c}L 2 \\
\text { (Engine Bay) }\end{array}$ & $\begin{array}{c}\text { (Cabin space) } \\
\text { (Luggage area) }\end{array}$ \\
\hline 0.2 & 0.6 & 0.2 \\
\hline
\end{tabular}

Table 2. CPD table for node E.

\begin{tabular}{cccc}
\hline $\operatorname{Pr}(E \mid L)$ & $\begin{array}{c}E 1 \\
(0.5-10 \\
\mathrm{MHz})\end{array}$ & $\begin{array}{c}E 2 \\
(10-100 \\
\mathrm{MHz})\end{array}$ & $\begin{array}{c}E 3 \\
(0.1-1 \\
\mathrm{GHz})\end{array}$ \\
\hline$L 1$ & 0.02 & 0.49 & 0.49 \\
$L 2$ & 0.02 & 0.49 & 0.41 \\
$L 3$ & 0.33 & 0.33 & 0.33 \\
\hline
\end{tabular}

Table 3. CPD table for node T.

\begin{tabular}{cccc}
\hline $\operatorname{Pr}(T \mid L)$ & $\begin{array}{c}T 1 \\
\left(0-24{ }^{\circ} \mathrm{C}\right)\end{array}$ & $\begin{array}{c}T 2 \\
\left(24-52{ }^{\circ} \mathrm{C}\right)\end{array}$ & $\begin{array}{c}T 3 \\
\left(52-80{ }^{\circ} \mathrm{C}\right)\end{array}$ \\
\hline$L 1$ & 0.2 & 0.45 & 0.35 \\
$L 2$ & 0.9 & 0.09 & 0.01 \\
$L 3$ & 0.85 & 0.14 & 0.0 \\
\hline
\end{tabular}

Table 4. CPD table for node P.

\begin{tabular}{ccccl}
\hline \multicolumn{1}{c}{$\operatorname{Pr}(P \mid T, E)$} & $\begin{array}{c}P 1 \\
\text { (good) }\end{array}$ & $\begin{array}{c}P 2 \\
\text { (moderate) }\end{array}$ & $\begin{array}{c}P 3 \\
\text { (poor) }\end{array}$ \\
\hline & & & & \\
$T 1$ & $E 1$ & 0.852 & 0.147 & 0.0 \\
& $E 2$ & 0.027 & 0.143 & 0.830 \\
& $E 3$ & 0.390 & 0.529 & 0.081 \\
$T 2$ & $E 2$ & 1.0 & 0.0 & 0.0 \\
& $E 3$ & 0.031 & 0.045 & 0.924 \\
& $E 1$ & 0.897 & 0.089 & 0.013 \\
$T 3$ & $E 2$ & 0.511 & 0.489 & 0.0 \\
& $E 3$ & 0.946 & 0.661 & 0.304 \\
& & & 0.054 & 0.0
\end{tabular}

to EMI noise frequencies $(0.5-1000 \mathrm{MHz})$ and changing temperature conditions $\left(0-80^{\circ} \mathrm{C}\right)$ were determined from simulations implements using Cadence Virtuoso software Cadence (2021). The procedure for introducing EMI noise and temperature effects in these simulations is outlined in the Appendix.

\subsection{BN for Predictive Analysis}

The application of BN template models is used for predicting the performance of components V1 and V2 in three different system scenarios. For each scenario, the CPDs associated to the node variables of the BN is provided in charts as illustrated in Fig. 3. For the case study, the CPD tables for variables $L, E$ and $T$ are identical in the $\mathrm{BN}$ template models for V1 and V2. Nevertheless, the CPD associated with the performance variable $P$ is obtained from the simulation data. For the comparison, the CPD charts for both $\mathrm{V} 1$ and $\mathrm{V} 2$ are combined together in Fig. 3 for three scenarios, as outlined below.

\subsubsection{Scenario 1}

In the first scenario, it is assumed that no additional information or evidence for any of the variables used in the $\mathrm{BN}$ model is available to the safety analyst. Without any additional evidence, it is only possible to determine the marginal probabilities associated with each of the node variables. Applying the chain rule of $\mathrm{BN}$, the joint probability distribution (JPD) is given as:

$$
\begin{aligned}
\operatorname{Pr}(L, E, T, P)= & \operatorname{Pr}(L) \operatorname{Pr}(E \mid L) \\
& \operatorname{Pr}(T \mid L) \operatorname{Pr}(P \mid E, T)
\end{aligned}
$$

Using the variable elimination method for the JPD of BN given in (1), the marginal probabilities 


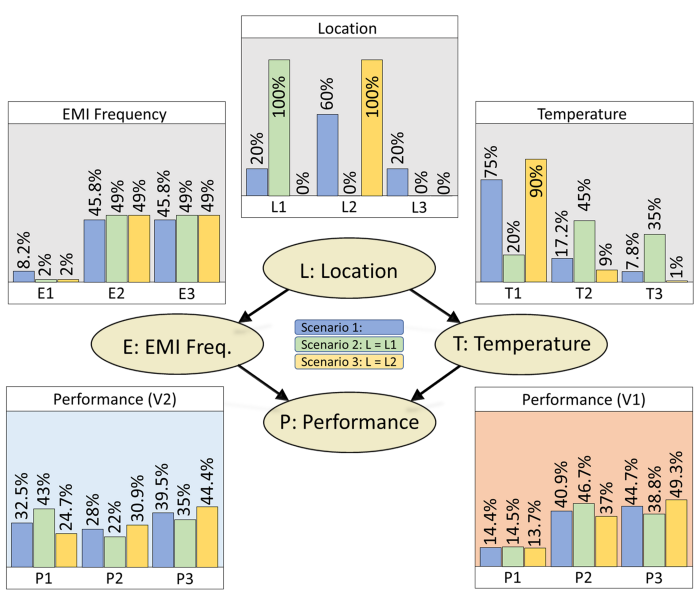

Fig. 3. BN template model used for predictive analysis, with 3 scenarios.

for each node variable in Fig. 3 may be calculated (see blue bars in CPD charts of Fig. 4). By comparing the performance charts for $\mathrm{V} 1$ and $\mathrm{V} 2$, we can see that the marginal probability, $\operatorname{Pr}(P 1$ :good $)$ is larger for component V2 than for V1. This comparison indicates that $\mathrm{V} 2$ would perform better than V1 in the presence of the epistemic uncertainty associated with EMI noise, temperature and location of the component within the system.

\subsubsection{Scenario $2-3$}

During the decision-making process, BN inference techniques could be used to answer queries under specific presumptions of evidence, as in Devaraj et al. (2021) and Norman and Martin (2004). For example, in scenario 2 , which assumes $L=L 1$, the performances of $\mathrm{V} 1$ and $\mathrm{V} 2$ can be compared on the assumption that the component would be located within the engine bay. A possible inference query associated with Scenario 2 could be, "What are the probabilities of good performance for components V1 and V2, when integrated within the engine bay". Using Bayes' theorem, the conditional probability for the above query is expressed as:

$$
\operatorname{Pr}(P 1 \mid L 1)=\frac{\operatorname{Pr}(L 1, E, T, P 1)}{\operatorname{Pr}(L 1, E, T, P)}
$$

To calculate such conditional probabilities, it is more convenient to use dedicated $\mathrm{BN}$ analysis software like the MSBN tool Kadie et al. (2001). By employing these tools, it is possible to assign a particular state for known variables as observed and instantly infer the probabilities of the unobserved variables in the BN. For Scenario 2 in Fig. 3. $L 1$ is assigned as the observed variable state of node $\mathrm{L}$ and the corresponding probability of

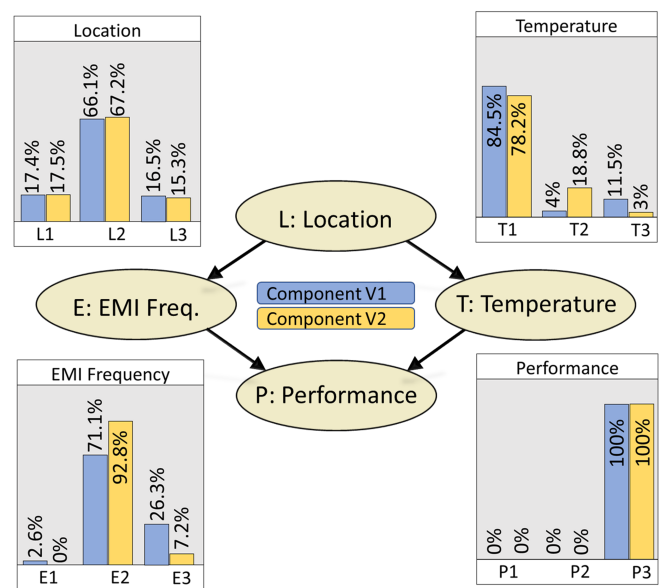

Fig. 4. BN template model used for diagnostic analysis for component V1 and V2.

good performance, $\operatorname{Pr}(P 1 \mid L 1)$ for $\mathrm{V} 2$ is found to be $43 \%$, whereas for $\mathrm{V} 1$, the probability of good performance is found to be $14.5 \%$. With the assumption of observed evidence on the component location, the probability of V2's performance to be good has improved by $10 \%$, but no change is seen for V1.

Similarly, for Scenario 3, L2 is assigned as the observed variable state of node $\mathrm{L}$ and thus, the probabilities of good performance for V1 and V2 when placed in the cabin are found to be $13.7 \%$ and $24.7 \%$, respectively. Comparing Scenarios 2 and 3 , it can be seen that the probability of achieving good performance from both components is higher when placed in the engine bay, instead of the cabin, although only marginally so for V1.

\subsection{BN for Diagnostic Analysis}

In Sub-section 4.1 examples of estimating probabilities in a $\mathrm{BN}$ by propagating information from cause to effect type scenarios were discussed. However, BNs also allow backward propagation of information for making predictions (i.e. effect to cause type inferences). In Fig. 4, the BN template model is used for a diagnostic analysis, where the probabilities associated with causal factors leading to a poor performance $(P 3)$ of components V1 and V2 is investigated. From the charts of Fig. 4, we can identify the causal factors or the variable states with the highest probabilities for making the performance of components V1 and V2 poor. For example, the worst-case would be the combination of EMI noise with frequencies in the range $10-100 \mathrm{MHz}(E 2)$, temperatures of 0-24 ${ }^{\circ} \mathrm{C}(T 1)$, and location in the cabin ( $\left.L 2\right)$. Based on this analysis, to avoid poor performance the following risk mitigation measures could be considered: 
- choose the engine bay or luggage compartment as the component location within the system;

- if the component is placed in the cabin, filters would be required to remove the EMI noise frequencies in the range $10-100 \mathrm{MHz}$ and the temperature would need to be maintained above $24{ }^{\circ} \mathrm{C}$.

\section{BN Templates for Collective Inference}

Template models can also be used for collective inference at system level, in cases where the likelihood of system malfunction depends on the performance of several components involved in the system function. For example, if a system consists of $n$ components and $k$ functions, then the performance of each component (denoted by nodes $P_{n}$ in Fig. 5p obtained from the template model can be used to determine the likelihood of $k^{t h}$ system malfunction (denoted by nodes $M_{k}$ in Fig. 5).

It is important to note that the components involved in performing a system function can be functionally dependent on each other. In such cases the influence of one component on the other need to be included with appropriate edges in the BN model, as shown in Fig. 5.(b).

\section{Conclusions}

Using a simple template BN model, the performance of two options for a safety-related component were analysed and compared using the case study presented in this paper. The examples discussed demonstrate the application of BN models in a knowledge-based approach for:

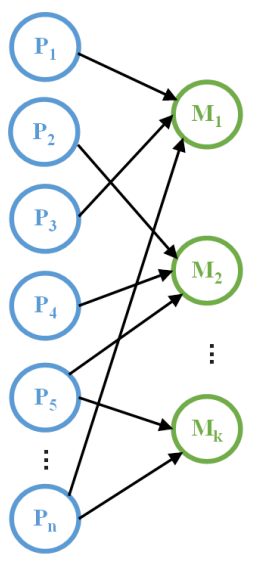

(a)

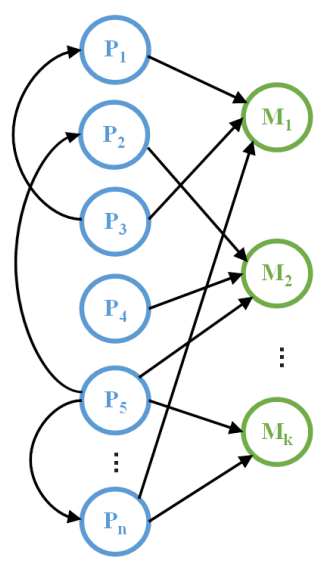

(b)
Fig. 5. Example to illustrate the use of BN template models to determine likelihood of system malfunction; a) without and b) with the consideration of functional dependence between components.
- visualizing various epistemic uncertainties in a single graphical model;

- combining evidence including both subjective beliefs and objective data to support likelihood estimation;

- applying BN inference techniques for predictive and diagnostic safety-analysis during the decision-making process;

- identifying the best-case and worst-case system safety conditions with quantifiable likelihood values;

- facilitating easier and more effective communication between vehicle manufacturers and their component suppliers regarding safety requirements, goals, and arguments.

For the adoption of a risk-based approach for EMC in relation to functional safety, graphical methods such as BNs can be efficient tools to model epistemic uncertainties and to make more informed decisions. Moreover, BN-based template models applied to estimate component performance could also be further extended for collective inference, such as to analyse their contribution to the performance of system-level functions.

\section{Appendix A. Component Simulations}

During the simulations, a number of temperature samples $(I=21)$, and EMI noise frequency samples $(J=96)$ were taken from ranges specified by states of variables $T$ and $E$ in the template. The samples were taken with a fixed step size within each range. A total of $I J=2016$ samples were simulated for each of the circuits V1 and V2. Parametric simulations of the performance characteristics for the circuits V1 and V2, under each combination of temperature $\left(0-80{ }^{\circ} \mathrm{C}\right)$ and EMI noise frequency $(0.5-1000 \mathrm{MHz})$, were carried out using the Spectre tool, Cadence (2021).

The impact of EMI noise was simulated by injecting a sinusoidal signal superimposed over the $5 \mathrm{~V}$ DC voltage in the supply power rails. The peak-to-peak amplitude of all EMI noise waveforms were kept at $1 \mathrm{~V}$ (i.e. $20 \%$ of the nominal input voltage). For each of the 2016 samples, the output frequency for V1 and V2 were recorded over the transient response time of 40 ns (see Fig. 6). Under nominal conditions the VCOs would require this transient response time to stabilize at their targeted operating frequencies.

For each simulation, the mean $(x)$ and the standard deviation $(\sigma)$ of the sampling points (output frequency values) over the transient time was obtained. The simulation data were used to calculate the maximum relative deviations $(D)$ from the nominal output frequency value $(N)$ using $(\mathrm{A} .1$, which were then classified using (A.2), where:

$$
D=\max \left[100\left|\frac{(x \pm 2 \sigma))-N}{N}\right|\right](\%)
$$




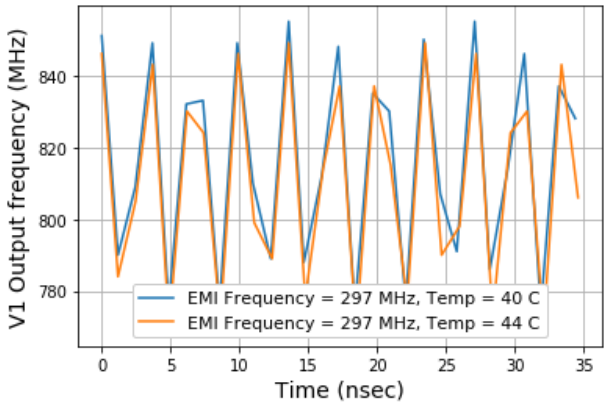

Fig. 6. Output from Spectre circuit simulation of V1 for a particular EMI noise frequency under varying ambient temperature conditions

$$
P= \begin{cases}P 1 & \text { if } 0 \leq D \leq 5 \\ P 2 & \text { if } 5<D \leq 10 \\ P 3 & \text { if } D>10\end{cases}
$$

\section{Acknowledgement}

The research leading to these results received funding from the European Union's Horizon 2020 research and innovation programme under the Marie SkłodowskaCurie grant agreement No 812790 (MSCA-ETN PETER - Pan-European Training, research and education network on Electromagnetic Risk management). This publication reflects only the authors' view, exempting the European Union from any liability. Project website: http://etn-peter.eu/.

\section{References}

Armstrong, K. (2006). Why EMC testing is inadequate for functional safety - and what should be done instead. In Proc. 1st IET International Conference on System Safety, pp. 179-183. London, UK.

Cadence (2021). Spectre Simulation Platform. https : //wwW.cadence.com/ko_KR/home/tools/ custom-ic-analog-rf-design/ circuit-simulation/ spectre-simulation-platform.html Accessed: 2021-04-12.

Devaraj, L., A. R. Ruddle, and A. P. Duffy (2020). System level risk analysis for immunity in automotive functional safety analyses. In Proc. 2020 International Symposium on Electromagnetic Compatibility (EMC Europe 2020). Rome, Italy.

Devaraj, L., A. R. Ruddle, A. P. Duffy, and A. J. M. Martin (2021). Risk-based approach for em resilience in complex systems using Bayesian Networks. In Proc. 2021 Joint IEEE International
Symposium on Electromagnetic Compatibility, Signal and Power integrity (EMC Europe 2021). Rome, Italy.

Fenton, N. and M. Neil (2007). Managing risk in the modern world applications of Bayesian networks. A Knowledge Transfer Report from the London Mathematical Society and the Knowledge Transfer Network for Industrial Mathematics. London Mathematical Society, London, UK.

Hanninen, M. and P. Kujala (2012). Influences of variables on ship collision probability in a Bayesian belief network model. Reliability Engineering and System Safety 102, 27-40.

Harima, K. et al. (2016). Experimental estimation of Efield distribution in a vehicle under multipath propagation environment using a reverberation chamber. In Proc. 2016 International Symposium on Antennas and Propagation (ISAP 2016). Okinawa, Japan.

Kadie, C. M., D. Hovel, and E. Horvitz (2001). MSBNx: a component-centric toolkit for modeling and inference with Bayesian Networks. Technical Report MSR-TR-2001-67, Microsoft Research, Redmond, WA 98052.

Koller, D. and N. Friedman (2007). Probabilistic Graphical Models: Principles and Techniques. MIT Press, Cambridge, MA.

Marquez, D., M. Neil, and N. Fenton (2010). Improved reliability modeling using Bayesian networks and dynamic discretization. Reliability Engineering System Safety 95, 412-425.

Norman, F. and N. Martin (2004). Combining evidence in risk analysis using Bayesian networks. Safety Critical Systems Newsletter 14, 8-13.

Ruddle, A. R. et al. (2004). Use of whole vehicle electromagnetic modelling in support of automotive EMC engineering. In Proc. 6th International European Symposium on Electromagnetic Compatibility (EMC Europe 2004), pp. 815-820. Eindhoven, Netherlands.

SERENE (1999). The SERENE Method Manual Version 1.0(F). Task 5.3 Report, EC Project No. 22187 (SERENE - SafEty and Risk Evaluation using bayesian NEts).

Washington, A. et al. (2019). Adoption of a Bayesian Belief Network for the system safety assessment of remotely piloted aircraft systems. Safety Science 118, 654-673. 